\title{
The Carrier Mobility in Organic Molecular Crystals
}

\author{
Zhang Ao \\ Beijing Institute of Graphic Communication \\ Beijing, China \\ email: ahf200805@sina.com
}

\author{
Zhang Chunxiu \\ Beijing Institute of Graphic Communication \\ Beijing, China
}

\author{
Pu Jialing \\ Beijing Institute of Graphic Communication \\ Beijing, China \\ e-mail: pujialing@bigc.edu.cn
}

\begin{abstract}
The exciton-phonon coupling is an very important process which determines the carrier mobility in organic crystals. By making some assumptions, the Hamiltonian be validate for organic molecular crystals. In the paper, the theory of carrier transport is extended to take weak quadratic coupling into account, and the diffusion constant be attained for a simple model. From the quadratic exciton-phonon coupling, we can get the analytical solution of carrier mobility in the organic molecular crystals. Experimentally, carrier mobility can be determined by various techniques.
\end{abstract}

\section{Keywords- carrier mobility, Hamiltonian, organic molecular}

\section{INTRODUCTION}

The carrier transport in organic crystals depends strongly on the exciton-phonon coupling. The carrier transport is studied for simultaneous local and nonlocal linear electron-phonon coupling[1-3]. Nonlocal exciton-phonon coupling increases the scattering, which gives lower the band contributions and higher hopping contributions [4]. It gives a phonon-assisted term which dominates at high temperature, leading to a constant diffusion coefficient whose magnitude depends on the ratio of the nonlocal to local coupling and is independent of transfer integral [4]. It changes the vibrations when an electron-hole pair is transferred between sites [4].

It is usually considered as a vibrational variation of the transfer integral between molecules, which gives a phonon-assisted term which dominates at higher temperature [5]. The study of carrier transport in organic material has a long history about 60 years when the semiconducting nature of organic crystals, their photoconductivity, and carrier mobilities were studied in these systems [5]. The most detailed transport theories are based on elaborations of the one-dimension Holstein molecular model. Phenomenological theories, including band theory and the polaron effective mass approach, have been successfully applied in a number of instances [5].
Local electron-phonon coupling changes the vibrations at a site where an electron-hole pair is created. For linear exciton-phonon coupling, the equilibrium position changes but the frequency does not change [6]. Grover and silbey assume a linear coupling of phonon and exciton. This approach is similar in spirit to the work on the small polaron [6].

An improved general theory of electronic transport in molecular crystal with local linear electron-phonon coupling is given. The electronic and phonon densities of states are taken as Gaussian type. The diffusion coefficient depends on temperature and the other parameters. The calculation provides a rational interpretation of the trends in the magnitude and temperature dependence of carrier mobilities in organic molecular crystals [7-14].

In Sec.II the definition for carrier mobility is written by the diffusion coefficient. In Sec.III the model Hamiltonian is presented for the carrier transport. In Sec.IV the carrier mobility without external field is given. In Sec.V it is the discussion of this paper.

\section{Characterization of CARRIER Mobility}

There are two categories for organic semiconductors : small molecules or oligomers and polymers. For most instances, this distinction does not rely on the actual ability the material to transport charges but rather reflects the ease of charge injection from electrodes usually used in devices [5].

Exciton transport in organic molecular crystals depends strongly on the exciton-phonon coupling. The carrier mobility is the most important quantity which characterizes charge transport. By the diffusion coefficient, the carrier mobility without an application of the external field is written [5]:

$$
\mu=\frac{e D}{k_{B} T}
$$


Here $k_{B}$ is the Boltzmann constant, e is the electron charge, $\mathrm{T}$ is the absolute temperature and $\mathrm{D}$ is the diffusion coefficient of carrier.

An external electric field induces a drift of the charge carriers. The mobility can be defined:

$$
\mu=\frac{v}{F}
$$

Here $v$ is the velocity of carrier and $\mathrm{F}$ is the amplitude of the applied electric field.

Diffusion should be regarded as a local displacement of the charge around an average position, while drift electron induces a displacement of the average position. Drift is the effect that dominates the migration of the charge across the charges across an organic layer in the devices. The carrier mobility is usually expressed in $\mathrm{cm}^{2} / V \cdot S$ [5].

According to perturbative microscopic models, the total mobility can be expressed to a good approximation as a sum of two contributions:

$$
\mu=\mu_{\text {tun }}+\mu_{\text {hop }}
$$

Where the first term is due to carrier tunneling and dominates transport at low temperature, and the second term is due to hopping motion and becomes dominant at higher temperature. The relative contributions of each mechanism depend on the actual values of the microscope parameters(electron-phonon coupling, electron and phonon bandwidths, and phonon energy) [5].

In the "Charge Transport in Organic Semiconductors", the forms of $\mu_{h o p}$ and $\mu_{\text {tun }}$ have been attained. In this case equal to $E_{p o l}=\hbar \omega_{0} g^{2}$, the tunneling mobility $\mu_{h o p}$ is given by [5]:

$$
\begin{gathered}
\mu_{h o p}=\frac{e a^{2} t^{2}}{k_{B} T \hbar^{2} \omega_{0}}\left[\frac{\pi}{g^{2} \operatorname{csch}\left(\hbar \omega_{0} / 2 k_{B} T\right)}\right]^{1 / 2} \\
\quad \times \exp \left[-2 g^{2} \tanh \left(\frac{\hbar \omega_{0}}{4 k_{B} T}\right)\right]
\end{gathered}
$$

Here $E_{p o l}$ is the polaron binding energy, and results from the deformations in molecular and lattice geometries that occur as the carrier localizes on a given site. $E_{p o l}$ has the from:

$$
E_{p o l}=\frac{1}{N} \sum_{q, j} \hbar \omega_{q, j}\left|g_{m}(q, j)\right|^{2}
$$

In the classical limit case for $\hbar \omega_{0}<<k_{B} T$, the tunneling mobility $\mu_{h o p}$ is given by [5]:

$$
\begin{gathered}
\mu_{\text {hop }}=\frac{e a^{2} t^{2}}{k_{B} T \hbar}\left[\frac{\pi}{2 E_{p o l} k_{B} T}\right]^{1 / 2} \\
\times \exp \left(-\frac{E_{p o l}}{2 k_{B} T}\right)
\end{gathered}
$$

In the narrow band limit, all band states are equally populated. As a result, the hopping mobility $\mu_{t u n}$ is written by [5]:

$$
\begin{aligned}
\mu_{t u n}= & \frac{e a^{2} \omega_{0}}{k_{B} T}\left[\frac{g^{2} \operatorname{csch}\left(\frac{\hbar \omega_{0}}{2 k_{B} T}\right)}{\pi}\right]^{1 / 2} \\
& \times \exp \left[-2 g^{2} \operatorname{csch}\left(\frac{\hbar \omega_{0}}{2 k_{B} T}\right)\right]
\end{aligned}
$$

The eq.7 is usually used to study the coherent part of the carrier mobility at very low temperature. It is noted that the expression does not depend on $\mathrm{t}$ [5].

The eq. 4 , eq. 6 and eq. 7 are agree with experiment, but the transport theories which based on the Holstein molecular model are difficult for the charge transport mechanisms in organic molecular crystals. In organic crystals, it is important that the variations in the transfer integral of acoustic and optical phonons are considered.

\section{HAMILTONIAN.}

When we consider for linearly and quadratically exciton-phonon interactions, the Hamiltonian for one exciton band and one phononband is written [6]:

$$
\begin{array}{r}
H=\sum_{n} \varepsilon a_{n}^{+} a_{n}+\sum_{n, m} J_{n m} a_{n}^{+} a_{m} \\
+\sum_{n} \omega\left(b_{n}^{+} b_{n}+\frac{1}{2}\right)+\sum_{n, m} K_{n m} b_{n}^{+} b_{m} \\
+\sum_{n, m} G_{n m} a_{n}^{+} a_{n}\left(b_{m}^{+}+b_{m}\right) \\
+g \sum_{n} a_{n}^{+} a_{n}\left(b_{n}^{+}+b_{n}\right)^{2}
\end{array}
$$

Here $\mathcal{E}$ is energy of an exciton at site n, $J_{n m}$ is the transfer integral between sites $\mathrm{n}$ and $\mathrm{m}$, and $\omega$ is the frequency of a phonon on site n. $G_{n m}$ is the linear coupling constant. $g$ is the quadratic coupling constant. The last two terms are the linearly and quadratically exciton-phonon interactions, respectively.

The Hamiltonian in wave vector is written [6]: 


$$
\begin{array}{r}
H=\sum_{k} \varepsilon_{k} a_{k}^{+} a_{k}+\sum_{\lambda} \omega_{\lambda}\left(b_{\lambda}^{+} b_{\lambda}+\frac{1}{2}\right) \\
+\frac{1}{N^{\frac{1}{2}}} \sum_{k, \lambda} \hat{G} a_{k+\lambda}^{+} a_{k}\left(b_{\lambda}+b_{-\lambda}^{+}\right) \\
+\frac{1}{N} \sum_{k, \lambda, \lambda^{\prime}} g a_{k+\lambda+\lambda^{\prime}}^{+} a_{k}\left(b_{\lambda}+b_{-\lambda}^{+}\right) \\
\times\left(b_{\lambda^{\prime}}+b_{-\lambda^{\prime}}^{+}\right)
\end{array}
$$

where

$$
\begin{gathered}
\mathcal{E}_{k}=\mathcal{E}+\sum_{n} J_{n m} e^{i k \cdot(n-m)}, \\
\omega_{k}=\omega+\sum_{n} K_{n m} e^{i \lambda \cdot(n-m)}, \\
\hat{G}_{\lambda}=\frac{1}{N} \sum_{n} G_{n m} e^{i \lambda \cdot(n-m)}
\end{gathered}
$$

In these equations $a_{n}^{+}$and $a_{n}$ are the creation and destruction operator for an exciton on site n, respectively. $b_{n}^{+}$and $b_{n}$ are the creation and destruction operator for an phonon on site, respectively. We have make assumption that the contributions of the nonlocal exciton-phonon coupling are neglected.

The linear coupling constant $G_{n m}$ is proportional to the charge of the energy of an exciton on site $\mathrm{n}$ when the phonon coordinate on site $\mathrm{m}$ is changed [6].

\section{The CARRIER Mobility Without EXTERnAL FIELD.}

By making three basic assumptions, we can get the diffusion coefficeint. The basic assumptions of the calculation are as follow: firstly, the exciton-phonon coupling is pure quadratic; secondly, we can use perturbation theory; thirdly, the exciton bandwidth is smaller than the phonon width. We get the diffusion coefficient [6]:

$$
\begin{aligned}
D & =\frac{\tilde{J}^{2}}{\Delta}\left(y^{2}+\frac{\Delta^{2}}{4 y \omega^{2}}\right) \\
& =\frac{2 a^{2} J^{2}}{\Delta} e^{-4 \gamma^{2}} e^{-4 y}\left(y^{2}+\frac{\Delta^{2}}{4 y \omega^{2}}\right)
\end{aligned}
$$

Where $a$ is the lattice spacing, $\mathrm{J}$ is the exciton bandwidth and $\Delta$ is the phonon bandwidth. $\omega_{\text {is }}$ the frequency of phonon. Here

$$
y=2 \gamma^{2} v(v+1)
$$

$$
v=\frac{1}{e^{\omega / k_{B} T}-1}
$$

Here $V$ is the thermal average number of phonons [6].

Calculating the correlation functions is the core of the carrier transport in organic molecular crystals. Apart from $\tilde{J}$, the correlation functions are the only place where details of the phonons enter and temperature dependence arises. These calculations take the treatment beyond the phenomenological theory [6].

By the Eq. 1, Eq. 13 and Eq. 14, we get the form of the carrier mobility without an external field

$$
\begin{gathered}
\mu=\frac{2 e a^{2} J^{2}}{k_{B} T \Delta} e^{-4 \gamma^{2}} e^{-8 \gamma^{2} v(v+1)}\left[4 \gamma^{4} v^{2}(v+1)^{2}\right. \\
\left.+\frac{\Delta^{2}}{8 \gamma^{2} v(v+1) \omega^{2}}\right]
\end{gathered}
$$

As $T \rightarrow 0, \quad v, y \rightarrow 0$ and $\mu$ diverges because there is no scattering in organic molecular crystals. With the temperature increases, the carrier mobility goes through the minimum. It then increases to reach a maximum value, finally obtain the zero with the temperature.

\section{DISCUSSION}

In this paper, we obtain the carrier mobility without external field. Transport theory based on the original Holstein molecular model which cannot fully describe the charge transport mechanisms in organic molecular crystal [5]. It is a very important process that the electron-phonon coupling determines the mechanism of the carrier transport in organic molecular crystals. We consider for linearly and quadratically exciton-phonon interactions. Experimentally, carrier mobility can be obtained by various experimental techniques. For the quadratic exciton-phonon coupling, we can attain the analytical solution of carrier mobility in the organic molecular crystals.

\section{ACKNOWLEDGMENT}

This project was supported by financially supported By BIGC(Ea201016), PHR20090515.

\section{REFERENCES}

[1] Holstein, T. Ann. Phys. 1959, 8, 325.

[2] Holstein, T. Ann. Phys. 1959, 8, 343.

[3] Munn, R. W.; Silbey, R. J. Chem. Phys. 1985, 83, 1843.

[4] Munn, R. W.; Silbey, R. J. Chem. Phys. 1985, 83, 1854.

[5] Veaceslav Coropceanu, el. J. Chem. Phys. 2007, 107, 926.

[6] Munn, R. W.; Silbey, R. J. Chem. Phys. 1978, 68, 2439.

[7] Silbey, R; Munn, R. W. J. Chem. Phys. 1980, 72, 2763. 
[8] Richard P. Hemengert; Katja Lakatos-lindenberg; Robert M. Pearlstein. J. Chem. Phys. 1974, 60, 3271.

[9] Munn, R. W. J. Chem. Phys. 1973, 58, 3230.

[10] Munn, R. W; Silbey, R. J. Chem. Phys. 1970, 52, 57.
[11] Munn, R. W. J. Chem. Phys. 1970, 52, 64.

[12] Munn, R. W.; Silbey, R. J. Chem. Phys. 1970, 52, 6391.

[13] Munn, R. W.; Williams, D.F. J. Chem. Phys. 1973, 59, 1742.

[14] Luty.T; Munn, R. W. J. Chem. Phys. 1983, 80, 3321. 